ISSN: 2576-8875

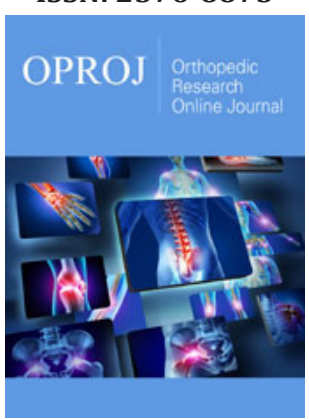

*Corresponding author: Sana Ullah, Department of Orthopedic and Spine Surgery Hayatabad Medical Complex Peshawar, Pakistan

Submission: 温September 14, 2021

Published: 酱 September 23, 2021

Volume 8 - Issue 5

How to cite this article: Sana Ullah, Muhammad Waqar, Waqas Haleem, et al. Outcomes of Arthroscopic Assisted Bankart Repair for Anterior Shoulder Instability Using Double Loaded All Suture Anchors Fixation. Ortho Res Online J. 8(5). OPROJ. 000698. 2021.

DOI: 10.31031/OPROJ.2021.08.000698

Copyright@: Sana Ullah, This article is distributed under the terms of the Creative Commons Attribution 4.0 International License, which permits unrestricted use and redistribution provided that the original author and source are credited.

\section{Outcomes of Arthroscopic Assisted Bankart Repair for Anterior Shoulder Instability Using Double Loaded All Suture Anchors Fixation}

\author{
Sana Ullah*, Muhammad Waqar, Waqas Haleem, Zeeshan Khan, Israr Ahmad \\ and Muhammad Arif Khan \\ Department of Orthopedic and Spine Surgery Hayatabad Medical Complex Peshawar, Pakistan
}

\begin{abstract}
The purpose of this study is to evaluate outcomes of Arthroscopic Bankart Repair (ABR) using double loaded All suture anchor technique, in young patients with posttraumatic Anterior Shoulder Instability (ASI).

Method: 24 patients were recruited who had traumatic anterior shoulder dislocation and underwent Arthroscopic Bankart Repair (ABR), with at least 2 years follow-up and minimum of 03 months of physiotherapy postoperatively. Recurrent instability, postoperative glenohumeral osteoarthritis, postsurgical complications and subjective scores (Simple Shoulder Test [SST], American Shoulder and Elbow Surgeon [ASES] and Rowe scores) were evaluated.
\end{abstract}

Results: Among the 24 participants, 03 patients (12.5\%) experienced at least one episode of redislocation, two patients (08\%) had signs and symptoms of glenohumeral osteoarthritis. The overall satisfaction rate was $80 \%$ with ASES, SST and Rowe scores of $82 \%, 9 \%$ and $79 \%$ respectively. One patient (04\%) had wound infection.

Conclusion: Arthroscopic Bankart Repair (ABR) using double loaded all- suture anchor fixation for anterior shoulder instability followed by physiotherapy has excellent short-term outcome in term of low post-surgical complication rate, high patient satisfaction, ability to join work back and improved subjective scores.

Keywords: Arthroscopic bankart repair; Anterior shoulder instability; Lebral repair

\section{Introduction}

Glenohumeral joint is a multiaxial ball and socket joint. The congruity of Glenohumeral joint is maintained by both static and dynamic stabilizers. Nature has scarificed the stability over mobility in shoulder. Abduction, extension and external rotation can dislocate the glenohumeral joint. In verity of sports and non-sports related injuries the Labrum and associated glenohumeral capsule/ligaments- the static stabilizers can be torn allowing the humrual head to dislocate from gelnoid. If the labrum and associated glenohumeral capsule/ ligaments does not heal or left untreated the shoulder continues to dislocate with minimal exertion and is said to be unstable. This dislocation has a negative effect on the functionality of the shoulder.

Bankart lesion is of two types i.e soft bankartin which anteroinferiorlabum avulse from gleniod rim and bony bankart which delineate anteroinferior glenoid rim fracture beside soft tissue injury. Patients with Bankart lesion experience shoulder pain that is diffuse in nature and gets worse with internal movement of shoulder. Dislocation of the shoulder joint occurs in 1 to $2 \%$ of the population [1]. Its incidence is $1.7 \%$ among adults and it is three times more common among men [2]. Ninety percent of shoulder dislocations are anterior [3] and traumatic injuries account for $95 \%$ of them [4-7]. In athletic patients under the age of 20 
years, the recurrence rates are greater than 90\% [4-7]. Among patients aged 20 to 25 years, the rates are between 50 and $75 \%$ [4-7].

In term of diagnosis, CT scan can't visualize detached labrum although to demonstrate labrum avulsion CT arthrograpgy has been used. CT scan can also show fracture around the glenoid. MRI is superior in detecting anterior glenoid labrum detachment plus bony fracture around the glenoid.

Traumatic labrum tear was first addressed by Bankart in 1923 and held it a reason behind Anterior Shoulder Instability (ASI). Over time different treatment strategies have been proposed for anterior shoulder instability including open and arthroscopic repairs, but in recent years the shift is more towards Arthroscopic repair. The open repair for Bankart lesion of anterior shoulder instability is still considered the gold standard by many surgeons $[8,9]$. But open repair does not always show us a significantly improvement of functionality of the shoulder. The initial results of arthroscopy were disappointing, with a recurrent rate of up to $49 \%$ using the Caspari'stransglenoidal suture, with the anchor technique, the results were better, but still higher than the open technique [10]. Due to technical advances combined with an increased understanding of the factors leading to recurrent instability, the results of Bankart arthroscopic repair have improved significantly and now can be compared with the results of the open technique [10]. Arthroscopic repair has the advantage of providing minimally invasive, anatomical reconstruction with low rates of operative complications and improved shoulder motion [11,12]. In absence of bone loss, Arthroscopic Bankart Repair (ABR) is presently the leading treatment modality [13-15]. The purpose of this study is to evaluate the results of Arthroscopic Bankart Repair (ABR) using suture anchor.

\section{Method}

We retrospectively reviewed our sports clinic record and recruited 24 patients (24 shoulders ) who underwent arthroscopic Bankart repair between Jan 2019 and july 2021 with minimum follow up for 2 years. All the surgeries were performed by a trained orthopedic and sports surgeon and the patients were followed by two orthopedic residents. All the included patients had age between 19 to 35 years. These patients had history of sport or nonsport related trauma to shoulder with resulting anterior shoulder dislocation. All these patients had Anterior Shoulder Instability confirmed on history and physical examination. Furthermore, all patients had Bankart lesion confirmed on magnetic resonance imaging before surgery. All Patients who had posttraumatic anterior shoulder dislocation evident on x-rays and physical examination after the injury, resulting in unidirectional Anterior Shoulder Instability and isolated Bankart lesion confirmed on Magnetic Resonance Imaging (MRI), who underwent Arthroscopic bankart repair using suture anchor technique, under the age of 40 years and have an active life style, with no vascular or neurological deficit, who were ready to participate in research study and willing to be contacted on mobile phone, had no co-morbidities and must be fit for General anesthesia and surgery and who must have gone through complete sessions (4 months at least) physiotherapy and rehabilitation postoperatively were included while patients with Atraumatic shoulder dislocation or multidirectional shoulder instability, with Glenoid bone loss more than $25 \%$ evident on preoperative Computerized Tomography Scan (CT scan), having associated Rotator cuff tear, engaging Hill-Sachs lesion, whom chief complaint was pain with shoulder movement under load rather than instability, who opt for open bankart repair and who had posttraumatic Anterior shoulder Instability in Bilateral shoulders were excluded from the study.

All the surgeries were performed by a single surgeon using same arthroscopic operative techniques in all the patients. Preoperatively, in waiting room, interscalene nerve block was given to all the patients for post-operative pain control. After General Anesthesia while the patient in supine position the effected shoulder was examined for anterior, posterior, inferior or multidirectional instability and range of motion was evaluated. The patients were then placed in lateral decubitus position, with the trunk angled $60^{\circ}$ forward. All the bony prominences were padded. Preoperative antibiotic was given before giving entering the ports. Standard three arthroscopic portals were namely anterior, anterosuperolateral and posterior were used. Diagnostic arthroscopy was performed. A $30^{\circ}$ Arthroscopic prob was used to detach the labrum form the anterior gleniod. A bleeding bed was created along the glenoid neck using burr and RASP. Through the inferior cannula drill guide for $4.2 \mathrm{~mm}$ knotless suture anchor was introduced and pilot hole was drilled in 5 o'clock and 3 o'clock position on the rim of gleniod for suture anchor. Two knotless suture anchors were used. The anchor was secured too glenoid. One of the suture limbs was run through the capsule and labrum. Polypropylene suture was used for skin closure, shoulder sling and abduction pillow was placed. Interscalene catheter was removed on $3^{\text {rd }}$ postoperative day.

\section{Postoperative rehabilitation}

All the participants received rehabilitation similar to the protocol mentioned by Law et al. [16] in their study (Table 1).

\section{Table 1:}

\begin{tabular}{|c|c|}
\hline Day 1 & $\begin{array}{c}\text { Pendulum exercise } \\
\text { Day } 3 \text { onward }\end{array}$ \\
\hline $1^{\text {st }}$ week onward & $\begin{array}{c}\text { Allow Internal rotation and forward flexion as pain } \\
\text { tolerated }\end{array}$ \\
\hline $4^{\text {th }}$ week onward & External Rotation with $90^{\circ}$ abduction \\
\hline $6^{\text {th }}$ week onward & $\begin{array}{c}\text { To regain full range of motion and start } \\
\text { strengthening exercise }\end{array}$ \\
\hline 2 months & Weight training, allow non-contact sports \\
\hline $4^{\text {th }}$ month & Return to contact sports and throwing \\
\hline
\end{tabular}

\section{Outcome measures}

In the follow up period after surgery the patients were assessed for recurrent instability, glenohumeral arthritis in operated shoulder radiographs, Subjective assessment (including, Simple Shoulder Test (SST), American Shoulder and Elbow Surgeon score (ASES) and Rowe score.) and complications. 


\section{Results}

\section{Recurrent instability}

Postoperative recurrent instability was reported in 3 cases out of 24 patients $(12.5 \%)$ of which 2 patients (8\%) had experienced second episode of trauma to the operated shoulder with complete dislocation on $16^{\text {th }}$ and $20^{\text {th }}$ month of index surgery. The remaining 01 patient $(4 \%)$ were persistently experiencing instability in operated shoulder with at least 2 episodes of dislocation on $12^{\text {th }}$, $15^{\text {th }}$ and $16^{\text {th }}$ months from index surgery.

\section{Glenohumeral arthritis}

On follow up radiographs of operated shoulder, after 2 years from index surgery, 02 patients $(12.5 \%)$ out of 24 had developed signs and symptoms of glenohumeral arthritis. Glenohumeral arthritis showed association with age and length of time from initial injury until surgery. Both patients $(12.5 \%)$ had an age above 32 years.

\section{Subjective assessment}

The overall patient satisfaction rate was $80 \%$, the mean ASES score was $82 \%$ with $80 \%$ excellent and good scores. The mean Rowe score was $79 \%$ with $76 \%$ excellent and good scores. Mean SST was $9 \%$.

\section{Complications}

1 patient (04\%) had developed superficial wound infection that resolved after a course of oral antibiotics.

\section{Discussion}

Our study mainly focuses on outcome and complications linked with arthroscopic bankart repair surgery using knotless suture anchor. Our study found that among total 24 participants, only $02(12.5 \%)$ patients had recurrent dislocation. Among these 02 patients had recurrent trauma leading to complete dislocation while $3^{\text {rd }}$ patient had episodes of dislocation on $12^{\text {th }}, 15^{\text {th }}$ and $20^{\text {th }}$ month after surgery. Two participants from our study reported shoulder joint osteoarthritis after 2 years of surgery and 1 patient had wound infection. The mean post-surgical score of ASES was $76 \%$. The mean score of post-surgical instability measured with ROWE was $79 \%$. Mean score of simple shoulder test for functional limitation was $9 \%$.

As discussed by Milchteim et al. [17] in his study, he reported the recurrent rate of $6.4 \%$ which is almost half as revealed by our study. This conflict in the results may be due to different population and different suturing used. But on other hand this study also support our results as our study reported the satisfaction rate of $80 \%$ and ROWE score of $84 \%$ which is almost same as reported by fore mentioned author.

Another study which definitely supports our results is done by Michael et al. [18]. He reported the recurrent dislocation rate to be $10 \%$ which is almost same as found by our study. This similarity in results could be due to same suturing technique. Author further reported the mean ASES and ROWE score to be 92.8 and 85.0 which is a bit higher than ours. This difference in results could be due to different population as this study was done on athletic population.

Similarly, Ono et al. [19] reported rate of osteoarthritis to be $36 \%$, which is higher than our results. This could be due to the greater follow-up time. On other hand, author reported the simple shoulder test score to be 10.8 which is almost same as ours results.

\section{Conclusion}

Arthroscopic Bankart Repair (ABR) using double loaded Allsuture anchor fixation for anterior shoulder instability followed by physiotherapy has excellent short-term outcome in term of low post-surgical complication rate, high patient satisfaction, ability to join work back and improved subjective scores.

\section{References}

1. Kazár B, Relovszky E (1969) Prognosis of primary dislocation of the shoulder. Acta Orthop Scand 40(2): 216-224.

2. Hovelius L (1982) Incidence of shoulder dislocation in Sweden. Clinical Orthopaedics and Related Research (166): 127-131.

3. Thomas PG (1988) Anterior glenohumeral instability. Orthopedics 11(1).

4. Henry JH, Genung JA (1982) Natural history of glenohumeral dislocationrevisited. Am J Sports Med 10(3): 135-137.

5. Hovelius L (1987) Anterior dislocation of the shoulder in teen-agers and young adults. Five-year prognosis. The Journal of Bone and Joint Surgery 69(3): 393-399.

6. Hovelius L (1978) Shoulder dislocation in Swedish ice hockey players. Am J Sports Med 6(6): 373-377.

7. Hovelius L, Augustini B, Fredin H (1996) Primary anterior dislocation of the shoulder in young patients. A ten-year prospective study. J Bone Joint Surg Am 78(11): 167716-84.

8. Bankart ASB (1938) The pathology and treatment of recurrent dislocation of the shoulder-joint. Br J Surg 26(101): 23-29.

9. Gummesson C, Atroshi I, Ekdahl C (2003) The disabilities of the arm, shoulder and hand (DASH) outcome questionnaire: Longitudinal construct validity and measuring self-rated health change after surgery. BMC Musculoskelet Disord 4: 1-6.

10. Lech O, Piluski P, Castillo C, Zanella D, Jara L (2017) Surgical repair of bankart lesion in recurrent shoulder dislocation: A comparative and retrospective study between open and arthroscopic technique.

11. Murphy A, Hurley E (2019) Long-term outcomes of the arthroscopic Bankart repair: A systematic review of studies at 10-year follow-up. J Shoulder Elbow Surg 28(11): 2084-208.

12. An V, Sivakumar B, Phan K (2016) A systematic review and meta-analysis of clinical and patient-reported outcomes following two procedures for recurrent traumatic anterior instability of the shoulder. Journal of Shoulder and Elbow Surgery 25(5): 853-863.

13. Marquardt B, Witt K, Liem D (2006) Arthroscopic bankart repair in traumatic anterior shoulder instability using a suture anchor technique. Arthroscopy 22(9): 931-936.

14. Mazzocca AD, Brown FM, Carreira DS, Hayden J, Romeo AA (2005) Arthroscopic anterior shoulder stabilization of collision and contract athletes. Am J Sports Med 33(1): 52-60.

15. Pulavarti RS, Symes TH, Rangan A (2009) Surgical interventions for anterior shoulder instability in adults. Cochrane Database of Systematic Reviews. 
16. Law BKY, Yung PSH, Ho EPY, Chang JJHT, Chan KM (2008) The surgical outcome of immediate arthroscopic Bankart repair for first time anterior shoulder dislocation in young active patients. Knee Surgery, Sport Traumatol Arthrosc 16(2): 188-193.

17. Milchteim C, Tucker SA, Nye DD, Lamour RJ, Liu W, et al. (2016) Outcomes of bankart repairs using modern arthroscopic technique in an athletic population. Arthroscopy 32(7): 1263-1270.
18. Saper MG, Milchteim C, Zondervan RL, Andrews JR, Ostrander RV (2017) Outcomes after arthroscopic bankart repair in adolescent athletes participating in collision and contact sports. Orthop J Sports Med 5(3).

19. Ono Y, Dávalos Herrera DA, Woodmass JM, Lemmex DB, Carroll MJ, et al. (2019) Long-term outcomes following isolated arthroscopic Bankart repair: A 9- to 12-year follow-up. JSES Open Access 3(3): 189-193.

For possible submissions Click below: 